\title{
POSITIVE SEQUENCE TOPOLOGICAL ENTROPY CHARACTERIZES CHAOTIC MAPS
}

\author{
N. FRANZOVÁ AND J. SMÍTAL
}

(Communicated by Andrew M. Bruckner)

\begin{abstract}
We prove that a continuous map $f$ of the interval is chaotic (in the sense of $\mathrm{Li}$ and Yorke) iff its sequence topological entropy $h_{A}(f)$ relative to a suitable increasing sequence $A$ of times is positive. This result is interesting since the ordinary topological entropy $h(f)$ of chaotic maps can be zero.
\end{abstract}

In the sequel, let $f$ be from the class $\mathrm{C}(I, I)$ of continuous maps $I \rightarrow I$, where $I$ is a compact real interval. The trajectory of $x \in I$ is a sequence $\left\{f^{n}(x)\right\}_{n=0}^{\infty}$ where $f^{n}$ denotes the $n$th iterate of $f$; the $\omega$-limit set of this trajectory is denoted by $\omega_{f}(x)$, and $\omega(f)=\bigcup\left\{\omega_{f}(x) ; x \in I\right\}$. A map $f$ is chaotic (in the sense of $\mathrm{Li}$ and Yorke) [6] if there is an uncountable set $S$ such that for any $x, y \in S, x \neq y$, and for any periodic point $p$ of $f, \limsup \left|f^{n}(x)-f^{n}(y)\right|>0, \liminf \left|f^{n}(x)-f^{n}(y)\right|=0$, and $\limsup \left|f^{n}(x)-f^{n}(p)\right|>0$, for $n \rightarrow \infty$; an extensive list of equivalent conditions, as well as other notions, terminology, and references can be found in [1].

Any map $f$ of positive topological entropy $h(f)$ is chaotic, but the converse is not true [6] (cf. also [1] for other references). It is interesting that contrary to this sequence topological entropy is a suitable tool for characterizing chaos. We prove the following theorem that completes results from [1].

Theorem. A map $f \in C(I, I)$ is chaotic in the sense of Li and Yorke iff there is an increasing sequence $A=\{n(i)\}_{i=1}^{\infty}$ of positive integers such that $h_{A}(f)$, the sequence topological entropy of $f$ with respect to $A$, is positive.

We recall the notion of metric sequence entropy, which is a generalization of Bowen's metric entropy. For compact metric spaces this entropy is equivalent to the topological sequence entropy [2].

Definition. Let $f \in C(I, I), A=\{n(i)\}_{i=1}^{\infty}$ be an increasing sequence of positive integers, $m>0$ be an integer, and $\varepsilon>0$. A set $E \subset I$ is an $(A, m, f, \varepsilon)$ span, if for any $x \in I$ there is some $y \in E$ such that $\left|f^{n(j)}(x)-f^{n(j)}(y)\right|<\varepsilon$

Received by the editors February 26, 1990.

1980 Mathematics Subject Classification (1985 Revision). Primary 26A18, 58F13, 58F11; Secondary $58 \mathrm{~F} 27,54 \mathrm{H} 20,28 \mathrm{D} 20$. 
for $1 \leq j \leq m$. Let $S(A, m, f, \varepsilon)$ be an $(A, m, f, \varepsilon)$-span with minimal possible number of points. Then

$$
h_{A}(f)=\lim _{\varepsilon \rightarrow 0} \limsup _{m \rightarrow \infty} \frac{1}{m} \log \operatorname{card} S(A, m, f, \varepsilon) .
$$

Remark. If $A$ is the set of positive integers then $h_{A}(f)=h(f)$ is (ordinary) topological entropy. There are examples proving that sequence entropy is a stronger invariant than ordinary entropy, and also examples showing that certain properties of ordinary topological entropy are not satisfied by the sequence entropy (cf. [2, 3], among others).

Proof of our theorem is based on the following

Lemma. Let $f \in C(I, I)$ be nonchaotic. Then for any $\varepsilon>0$ there are points $x_{1}, \ldots, x_{k} \in \omega(f)$, and a set $U \supset \omega(f)$, relatively open in $I$, with the following property: if

$$
f^{j}(x) \in U \text { for } 0 \leq j \leq r
$$

then there is some $i$ such that for any $j$ with $0 \leq j \leq r$,

$$
\left|f^{j}(x)-f^{j}\left(x_{i}\right)\right|<\varepsilon .
$$

Proof. Let $f$ be nonchaotic. Then by Theorem 2.3 in [1],

$$
f \mid \omega(f) \text { is Ljapunov stable (has equicontinuous powers) }
$$

and

$$
\text { every point } u \in \omega(f) \text { is almost periodic }
$$

(in some terminology also "regularly almost periodic"), for any neighborhood $G$ of $u$ there is an integer $n>0$ such that $f^{n j}(u) \in G$ for every $j$. Let $\varepsilon>0$. By (4), for any $u \in \omega(f)$ there is a neighborhood $O(u)$ of $u$, relatively open in $I$ such that

$$
\left|f^{j}(u)-f^{j}(v)\right|<\varepsilon / 2 \text { for any } v \in O(u) \cap \omega(f) \text { and any } j .
$$

Since $\omega(f)$ is compact (cf. [4]) there are points $x_{1}, \ldots, x_{k}$ from $\omega(f)$ such that $\omega(f) \subset 0\left(x_{1}\right) \cup \cdots \cup 0\left(x_{k}\right)$. By (5), for any $y \in 0\left(x_{i}\right) \cap \omega(f)$ there is a positive integer $q(y, i)$ such that $f^{q(y, i)}(y) \in 0\left(x_{i}\right)$; we can clearly assume that

$$
q(y, i) \text { is divisible by } q\left(x_{i}, i\right) \text { if } y \in 0\left(x_{i}\right) \cap \omega(f) .
$$

By the continuity of $f$, there is a neighborhood $U(y, i) \subset 0\left(x_{i}\right)$ of $y$ in $I$ such that

$$
f^{q(y, i)}(U(y, i)) \subset 0\left(x_{i}\right)
$$

and

$$
\operatorname{diam} f^{j}(U(y, i))<\varepsilon / 2 \text { for } 0 \leq j \leq q(y, i) .
$$


Put $U_{i}=\bigcup\left\{U(y, i) ; y \in 0\left(x_{i}\right) \cap \omega(f)\right\}$ and $U=U_{1} \cup \cdots \cup U_{k}$. Clearly $U$ is open in $I$ and $\omega(f) \subset U$.

Now assume (2) and fix some $j$. We prove (3). There are $y \in \omega(f)$ and $i$ such that $x \in U(y, i)$. By (8) and the well-known fact that $f(\omega(f))=$ $\omega(f)$, there is a maximal sequence $\{y(t)\}$ (finite or infinite) of points from $U_{i}$ such that $y(0)=y$ and $f^{p(n)}(x) \in U(y(n), i)$, where $p(0)=0$ and $p(n)=$ $q(y(0), i)+\cdots+q(y(n-1), i)$ for $n>0$. By (2) there is some $n$ with $p(n) \leq$ $j<p(n+1)$. Then $\left|f^{j}(x)-f^{j}\left(x_{i}\right)\right| \leq A+B$, where $A=\mid f^{j-p(n)}\left(f^{p(n)}(x)\right)-$ $f^{j-p(n)}(y(n)) \mid$ and $B=\left|f^{j-p(n)}(y(n))-f^{j-p(n)}\left(f^{p(n)}\left(x_{i}\right)\right)\right|$. Since $j-p(n)<$ $q(y(n), i),(9)$ implies $A<\varepsilon / 2$. Since $\omega(f)$ is invariant under $f$, by (7) we have $f^{p(n)}\left(x_{i}\right) \in 0\left(x_{i}\right) \cap \omega(f)$, hence (6) implies $B<\varepsilon / 2$. This proves (3).

Proof of Theorem. Let $f$ be nonchaotic and let $\varepsilon>0$. Let $U$ and $x_{i}$ be as in the lemma. Let $\left\{K_{i}\right\}_{i=1}^{s}$ be pairwise disjoint sets with $\operatorname{diam} K_{i}<\varepsilon$ for any $i$, and $K_{1} \cup \cdots \cup K_{s}=I \backslash U$. Assign to any $x \in I$ an itinerary $\alpha(x)=$ $\left\{\alpha_{j}(x)\right\}_{j=0}^{\infty}$ such that $\alpha_{j}(x)=K_{i}$ if $f^{j}(x) \in K_{i}$. If $f^{j}(x) \in U$, let $M(j)$ be the maximal subinterval of the set of nonnegative integers such that $j \in M(j)$ and $f^{i}(x) \in U$ for any $i \in M(j)$. Then by the lemma there is some $t$ such that $\left|f^{i}(x)-f^{i}\left(x_{t}\right)\right|<\varepsilon$ for any $i \in M(j)$. Put $\alpha_{i}(x)=t$ for any $i \in M(j)$.

It is easy to see that for any $x, y \in I, \alpha(x)=\alpha(y)$ implies $\left|f^{j}(x)-f^{j}(y)\right|<$ $2 \varepsilon$, for any $j$. Let $A=\{n(i)\}_{i=1}^{\infty}$ be an increasing sequence. Keeping the notation from the definition, we get card $S(A, m, f, 2 \varepsilon) \leq \operatorname{card} N(m)$, where $N(m)$ is the set of all possible codes $\left\{\alpha_{n(j)}(x)\right\}_{j=1}^{m}$. By Birkhoff's theorem (cf. Šarkovskii's refinement [5]), for any neighborhood $U$ of $\omega(f)$ there is an integer $q>0$ such that the number of points of an arbitrary trajectory lying outside $U$, is less than $q$. Consequently, every code from $N(m)$ consists of at most $2 q+1$ blocks, and each block is formed by only one of the symbols $1, \ldots, k, K_{1}, \ldots, K_{s}$ (with possible repetitions). It is easy to verify that card $N(m)=o\left(n^{2 q+2}\right)$, and (1) gives $h_{A}(f)=0$.

Now let $f$ be chaotic. We may assume that $h(f)=0$, since otherwise $h_{A}(f)=h(f)>0$, if $A$ is the set of positive integers. By [6] (cf. also Theorem 2.3 in [1]) there is an infinite $\omega$-limit set containing two nonseparable points $u<v$. Choose $\varepsilon$ with $0<\varepsilon<v-u$. For any integer $i>0$, let $X(i)$ be the set of $i$-tuples of binary symbols 0 and 1. By Lemma 4.1 in [6], there is a system $\left\{A_{\alpha}: \alpha \in X(i)\right\}_{i=1}^{\infty}$ of compact subintervals of $I$, and an increasing sequence $A=\{n(i)\}_{i=1}^{\infty}$ of positive integers such that, for any sufficiently large $i$,

$$
\begin{gathered}
A_{\alpha \beta} \subset A_{\alpha} \quad \text { if } \alpha \beta \in X(i), \\
A_{\alpha} \cap A_{\beta}=\varnothing \quad \text { if } \alpha, \beta \in X(i), \quad \alpha \neq \beta, \\
\operatorname{dist}\left(f^{n(i)}\left(A_{\alpha 0}\right), \quad f^{n(i)}\left(A_{\alpha 1}\right)\right)>\varepsilon \quad \text { if } \alpha \in X(i) .
\end{gathered}
$$

Here $\alpha \beta$ or $\alpha 0$ means concatenation of codes. Let $i \geq 1$ and choose $x_{\alpha} \in A_{\alpha}$ 
for every $\alpha \in X(i)$. If $\alpha, \beta \in X(i), \alpha \neq \beta$, then by (12), $\mid f^{n(j)}\left(x_{\alpha}\right)-$ $f^{n(j)}\left(x_{\beta}\right) \mid>\varepsilon$ for a suitable $j \leq i$. Let $M_{i}=\left\{x_{\alpha} ; \alpha \in X(i)\right\}$. Then by (10) and (11), $\operatorname{card} S(A, i, f, \varepsilon / 2) \geq \operatorname{card} M_{i}=2^{i}$, and by (1) we get $h_{A}(f) \geq$ $\log 2>0$.

Remark. Here we only need a part of Lemma 4.1 from [6]. An analysis of its proof and application of results from [1] shows that in the theorem we can take $A=\left\{2^{i}\right\}_{i=0}^{\infty}$; however, the proof does not seem to be easy.

\section{REFERENCES}

1. V. V. Fedorenko, A. N. Šarkovskii, and J. Smital, Characterizations of weakly chaotic maps of the interval, Proc. Amer. Math. Soc. 110 (1990), 141-148.

2. T. N. T. Goodman, Topological sequence entropy, Proc. London Math. Soc. (3) 29 (1974), 331-350.

3. M. Lemańczyk, The sequence entropy for Morse shifts and some counterexamples, Studia Math. 82 (1985), 221-241.

4. A. N. Sarkovskii, Nonwandering points and the center of a continuous map of the line into itself, Dopovidi Akad. Nauk Ukrain. RSR Ser. A. (1964), 865-868. (Ukrainian)

5. $\ldots$, On a theorem of G. Birkhoff, Dopovidi Akad. Nauk Ukrain. RSR. Ser. A 5 (1967), 429-432. (Ukrainian)

6. J. Smital, Chaotic functions with zero topological entropy, Trans. Amer. Math. Soc. 297 (1986), 269-282.

Institute of Mathematics, Comenius University, 84215 Bratislava, Czechoslovakia 